\title{
Partial Mole with a Coexistent Viable Fetus-A Clinical Dilemma: A Case Report with Review of Literature
}

\author{
${ }^{1}$ Archana D Rathod, ${ }^{2}$ SP Pajai, ${ }^{3}$ A Gaddikeri
}

\begin{abstract}
Gestational trophoblastic disease encompasses a diverse group of lesions. If molar changes in the placenta are known along with an alive fetus, then situation is difficult for both obstetrician and parents. On one hand, there may be a normal pregnancy whereas on the other hand the mother may be threatened by numerous complications caused by the hydatid mole, if the pregnancy is continued.

We present successfully managed case of partial molar pregnancy with an alive fetus at 1st stage of preterm labor with premature rupture of membranes with anemia with a live diploid female fetus with good neonatal out come. Follow-up till 1 year showed no progression to malignant gestational trophoblastic diseases.
\end{abstract}

Keywords: Partial molar pregnancy, Coexistent live fetus, Karyotyping, Maternal complications, Partial molar placenta, Normal fetus.

How to cite this article: Rathod AD, Pajai SP, Gaddikeri A. Partial Mole with a Coexistent Viable Fetus-A Clinical Dilemma: A Case Report with Review of Literature. J South Asian Feder Obst Gynae 2014;6(1):51-55.

Source of support: Nil

Conflict of interest: None

\section{INTRODUCTION}

Gestational trophoblastic disease consists of a broad spectrum of conditions ranging from an uncomplicated partial hydatidiform molar pregnancy to stage IV choriocarcinoma with cerebral metastasis.

Hydatidiform mole is characterized by abnormal fetoplacental development and trophoblastic hyperplasia, resulting from genetically abnormal conception when there is excessive paternally derived genetic material.

It is classified into complete mole when there is total replacement of normal placenta by grossly dilated and

\footnotetext{
${ }^{1}$ Assistant Professor, ${ }^{2}$ Professor and Head, ${ }^{3}$ Senior Resident

1-3Department of Obstetrics and Gynecology, Shri Vasantrao Naik Government Medical College, Yavatmal, Maharashtra India
}

Corresponding Author: Archana D Rathod, Assistant Professor Department of Obstetrics and Gynecology, Shri Vasantrao Naik Government Medical College, Yavatmal, Maharashtra, India Phone: 9823273422, e-mail: archanarathod7@gmail.com hydropic villi in the absence of fetus and the incomplete or partial mole showing partial replacement with hydropic villi and visible abnormal fetal parts leading to termination of the pregnancy in first trimester. Fetus in such cases is rarely alive at the time of diagnosis and often shows gross congenital anomalies associated with triploidy and have a grave prognosis due to limited functional placenta and severe intrauterine fetal growth retardation. ${ }^{1}$

Partial molar pregnancy with coexistent live fetus as seen in our case is extremely rare excluding cases of multiple conceptions. Incidence of a normal fetus and partial molar placenta is 0.005 to $0.01 \%$ of all pregnancies. ${ }^{2}$

Such association has been divided into three types. ${ }^{1}$

1. The first and most common is a twin pregnancy with one normal fetus having a normal placenta and another complete mole.

2. Second type is a twin pregnancy with normal fetus and placenta and another partial mole.

3. The third and most uncommon occurrence is a singleton normal fetus with partial molar placenta.

Such a fetus should have a normal karyotype to survive. Placenta in a partial mole with fetus in a singleton pregnancy results from dispermy and has a triploid karyotype in most cases. $^{3}$

Partial molar placenta with coexistent singleton normal fetus with normal karyotype has been reported only seventeen times in extensively searched medical literature. ${ }^{4}$

We present a case who was diagnosed to have partial hydatidiform mole along with live fetus at 22 weeks of gestation and was referred to us at 34 weeks of gestation in first stage of preterm labor with premature rupture of membranes with anemia.

\section{CASE REPORT}

A 23 years old lady, who was primigravida, was referred form nearby hospital for parital molar pregnancy with preterm labor with premature rupture of membranes with gestational amenorrhea of 34 weeks.

She was a primigravida with age of marriage of 1 year. During her antenatal period, she had her first ANC visit at 22 weeks of gestation at private gynecologist. She did not give history of vaginal bleeding, excessive vomiting and lower abdominal pain. 
Ultrasonographic examination at 22 weeks revealed a single live fetus with a huge multicystic placenta suggestive of diffuse molar change with no fetal structural abnormalities and fetal biometry was normal except abdominal circumference which was slightly less for gestational age (Fig. 1). Thyroid function test was within normal limits.

Even though no other significant abnormalities were noted during her first USG report, the risk of subsequent fetal abnormality and maternal complications like malignant transformation were explained to the patient and her family.

Due to religious beliefs regarding pregnancy termination, and as USG reports did not show any fetal abnormalities without any maternal complications, the patient and her family decided to continue her pregency with frequent and regular ANC follow-up. Serial obstetrical ultrasound were within normal limits except mild IUGR with thick placenta with multiple cysts suggestive of partial mole. She was on oral iron and calcium. Her blood group was $\mathrm{BRh}+$ ve and $\mathrm{Hb} \%$ was $7 \mathrm{gm} \%$.

There were no obstetric complications until the 32nd gestational week when patient developed preeclampsia and was put on tab. Nifedipine $10 \mathrm{mg}$ BD with regular blood pressure monitoring.

At 34 weeks, she had premature rupture of membranes with preterm labor and attended nearby hospital, but she was referred to us for further management and NICU care. She arrived in the emergency room with 6 hours of premature rupture of membranes. Her vitals were normal. On examination her uterine height was more than gestational age with normal fetal heart sounds with moderate degree of anemia $(\mathrm{Hb} \%-7 \mathrm{gm} \%$ ) serum $\beta$-hCG level was 23,500 MIU/1 at 34 weeks.

USG obstetric showed an alive fetus with breech presentation of average fetal maturity of 32 weeks with huge multicystic placenta of thickness $10.7 \mathrm{~cm}$ with multiple

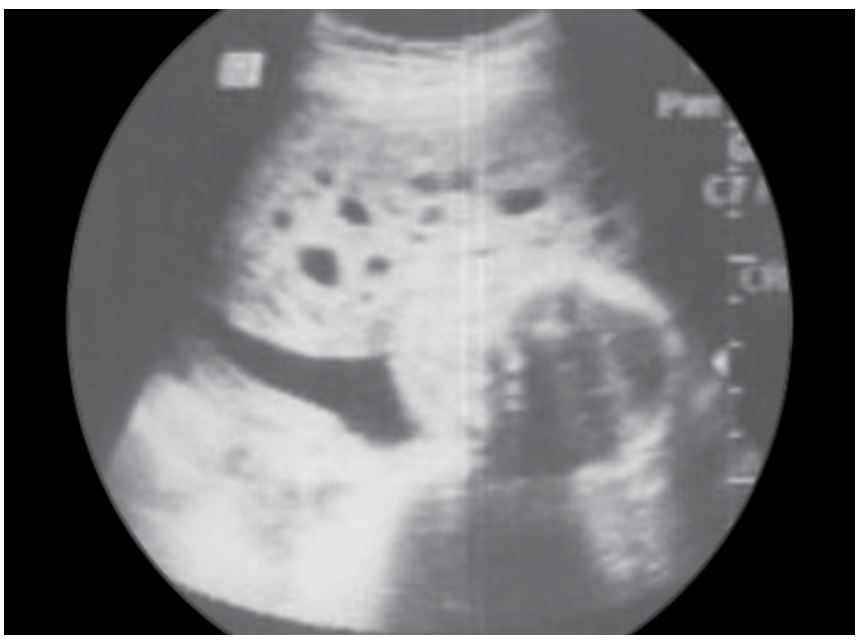

Fig. 1: USG picture at 22 weeks of gestation showing partial molar placenta with BPD cystic spaces with venous lake and normal thin placental tissue, suggestive of partial mole (Fig. 2).

Internal findings showed 3 to $4 \mathrm{~cm}$ dilated cervical so and membranes were absent with clear liquor. Intrapartum fetal surveillance was within normal limits. She transfused 1 unit of cross matched compatible blood.

After 6 hours, she delivered a female baby by breech vaginally of weight $1400 \mathrm{gm}$ with APGAR score of 9 and 10 at 1 and 5 minutes respectively.

Baby cried immediately after birth. Placenta was delivered by Brandt's Andrews method. Weight of placenta was 1700 gm with multiple grapes like cysts within it (Fig. 3). Active management of third stage of labor reduced the most anticipated complication of $\mathrm{PPH}$ in this patient.

Baby was admitted to NICU in view of prematurity. Baby's neurosonogram, USG abdomen, CVS study and fundus examination revealed normal. Baby had no major abnormality other than microcephaly $(\mathrm{HC}-28 \mathrm{~cm})$. Baby get discharged after 27 days with weight of $1700 \mathrm{gm}$.

Additional maternal investigations (RFT, LFT, CBC. Chest X-ray, coagulation profile) done after delivery were normal. Check USG scan done on 1st postpartum day showed no any retained placenta or molar tissue. Placenta was sent for histopathology examination.

The patient was being followed up weekly with urinary $\beta$-hCG test as we do not have facility of serum $\beta$-hCG in our institute. Urine pregnancy test was negative in 6 weeks postpartum and on her regular follow-up till 12 months. Repeat ultrasound did not show features of myometrial invasion. Postnatal period was uneventful.

The baby's growth was within normal limits till 1 year without any developmetal milestones delay. Karyotyping of the baby was done suggestive of diploid karyotype 46XX.

Histopathology report of the placenta confirmed diagnosis of partial mole showing an admixture of normal villi and molar villi with dilated cistern formation (Fig. 4).

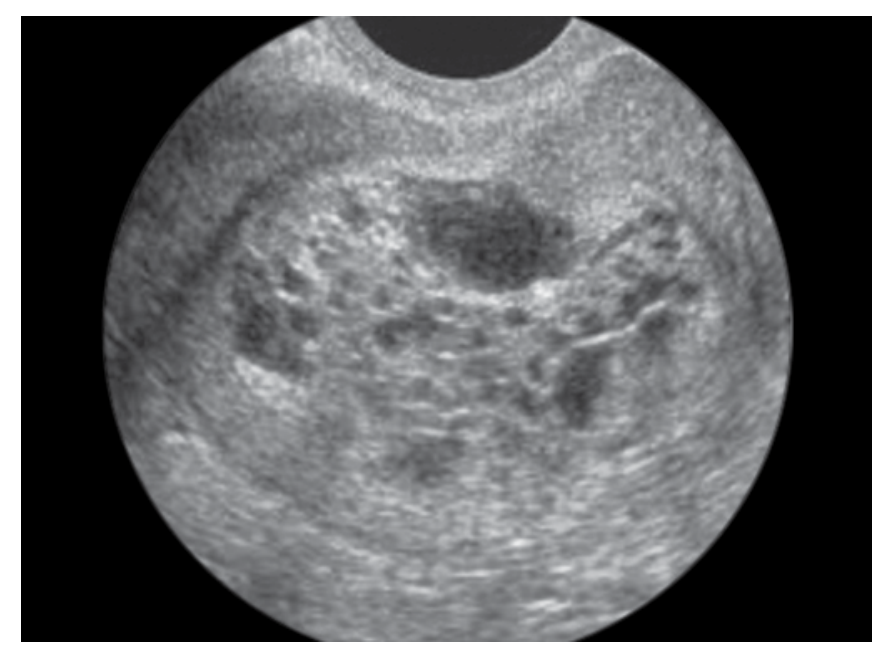

Fig. 2: USG picture of partial molar placenta with cysts 


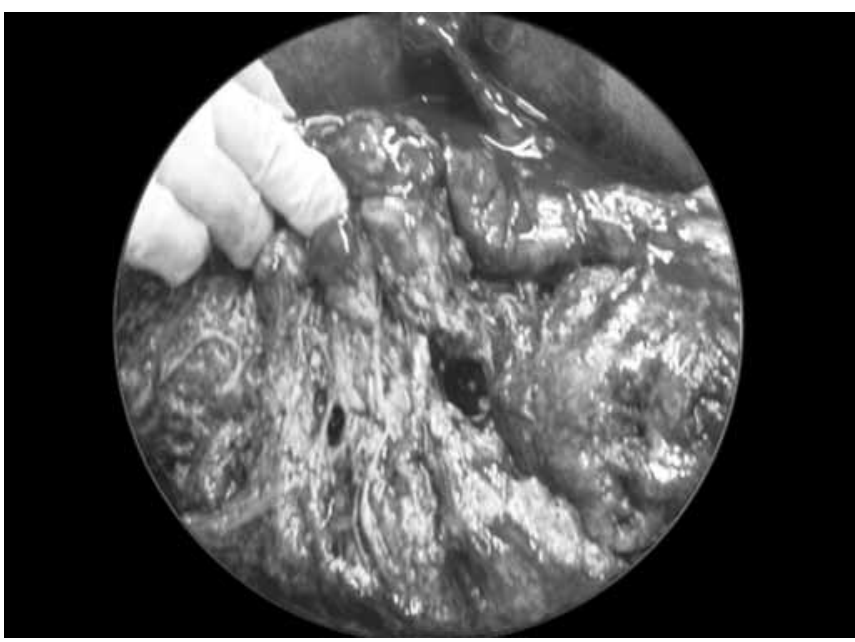

Fig. 3: Huge placenta with multiple cysts of weight $1.7 \mathrm{~kg}$

\section{DISCUSSION}

In case of a singleton normal fetus with partial molar placenta, fetal survival depends upon several factors.

1. Normal karyotype of the fetus (sarno et al 1993). ${ }^{5}$

2. Smaller molar placenta compared to normal placenta (Jones, and Lauersen, 1975, Deaten et al). ${ }^{6}$

3. The onset of molar degeneration and its speed of degeneration (Jones and Lauersan, 1975, Sarno et al, 1993). ${ }^{7}$

4. Absence of anemia occurring in the fetus (Crooij et al 1993). ${ }^{8}$

5. Absence of maternal complications such as pre-eclampsia, thyrotoxicosis and vaginal bleeding interrupting the pregnancy (Teng and Ballon, 1984). ${ }^{9}$

Szulman and Surti et al reported eight cases of partial mole coexisting with the fetus with normal karyotype after 15 gestational weeks, but only two of the infants survived. ${ }^{10}$

Agarwal R et al from AIIMS, New Delhi reported partial molar pregnancy with diploid live fetus, which terminated at 28 weeks with good neonatal outcome. ${ }^{2}$

Parveen $\mathrm{Z}$ et al reported a partial hydatidiform mole along with term gestation ended in normal alive and healthy fetus. ${ }^{11}$

Pool $\mathrm{R}$ et al reported partial mole with coexistent live full term fetus. The pregnancy proceeded to term and ended in the spontaneous vaginal delivery of a normal baby. The placenta showed areas of macroscopic cystic degeneration with the histological features of hydatiform mole. ${ }^{12}$ Kajal kiran Dhingra et al reported two cases of singleton pregnancy, in which focal placental molar change was deleted. However, the pregnancy ended in phenotypically normal term fetus. ${ }^{13}$

Vejerslev (1991) reviewed 113 reports of pregnancies with mole and fetus in which there appeared to be no major malformations or cytogenetic abnormalities of these, 87

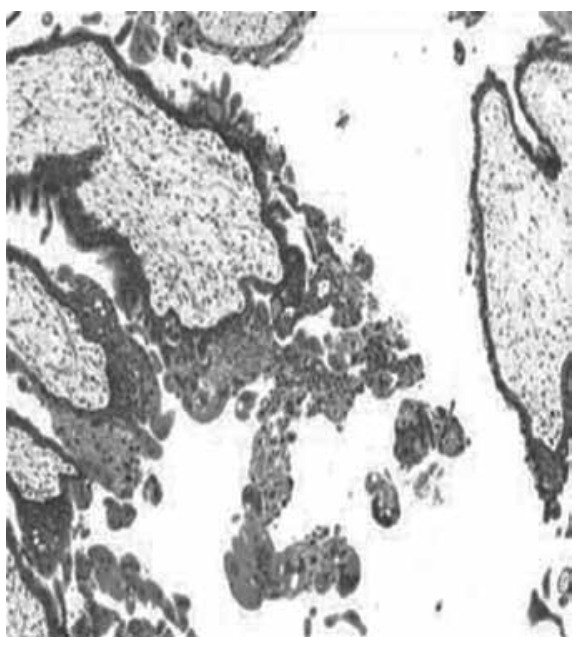

Fig. 4: Histopathological picture of the placenta showing an admixture of normal villi and molar villi with dilated cistern formation

had intended to continue the pregnancy with or without knowledge of accompanying mole, in fact 52 pregnancies $(59.8 \%)$ proceeded to the 28 th weeks and a risk for either substantial bleeding or pre-eclamptic symptoms developed in $\sim 30 \%$. In $\sim 15 \%$ the degree of severity required termination on clinical grounds irrespective of fetal week until birth ultrasonographic examination before and after delivery found no signs of congestive heart failure nor any other evidence of chronic fetal blood loss throughout development. Of pregnancies which continued beyond the 28th week, a surviving child may be expected in $\sim 70 \%$ of pregnancies, the risk of IUD or neonatal death being $\sim 30 \% .9 .1 \%$ of those developed persistent trophoblastic disease. ${ }^{14}$

The present case was followed sonographically from the 22nd gestational week until birth. Ultrasonographic examination before and after delivery found no signs of congestive heart failure nor any evidence of chronic fetal blood loss throughout the pregnancy.

\section{PREGNANCY}

Molar pregnancy with a coexisting fetus progressing to a viable, healthy fetus is an extreme rarity. In most of these cases, the pregnancy is terminated due to severe complications, such as vaginal bleeding, pre-eclampsia and missed abortion (Table 1). As a result, management of molar pregnancy with coexisting fetus remains controversial. In the present case, the decision of the patient to continue the pregnancy was accepted, not only because of good fetal development with no fetal abnormality with absence of maternal complications. But, we can not ignore high risk of developing persistent gestational trophoblastic disease. Therefore, proper prenatal consultation should consider both the outcome and possible complications of such a pregnancy. 
Table 1: Review of molar pregnancy with coexistent normal fetus and outcome

\begin{tabular}{llll}
\hline References & Diagnosis/delivery(gw) & Karyotype & Outcome \\
\hline Jones and Lauersen $(1975)^{6}$ & $40 / 40$ & NA & 2900 gm normal female \\
Crooiji et al (1985) & $27 / 27.3$ & 46 XX & Fetal distress with anemia, 980 gm, died \\
Ronald et al (1988) & $19 / 23.5$ & 46 XX & Polyhydramnios, PROM, 870 gm, died \\
Sarno et al (1993) & $13.9 / 33.6$ & 46 XX & 1935 gm, normal female fetus \\
Present case & $22 / 34$ & 46 XX & Pre-eclampsia, PROM, 1400 gm, female \\
& & & fetus \\
\hline
\end{tabular}

Nwosu et al reported that no reports demonstrate that if pregnancy is kept to term, it will increase the incidence of invasive mole or choriocarcinoma. ${ }^{15}$

Zahida P et al from Pakistan, reported G4 P1 + 2 lady with partial mole along with alive baby managed conservatively 18 weeks onward and delivered successfully at term. ${ }^{16}$

Hsieh CC et al from Department of Obstetrics and Gynecology, Taiwan, Republic of China, reported delivery of a severely anemic fetus after partial molar pregnancy. ${ }^{1}$

Although anemia is one of the risk factors that jeopardize the fetus in the case of partial molar pregnancy, termination is not indicated when the fetus is normal and no complications have occurred.

Khoo SK et al reviewed 24 patients with a molar placenta and coexisting live fetus (1965-1985). The rate of fetal abnormalities was $33 \%$ with chromosomal karyotype was triploidy 69XXX. In 3 patients malignant sequel were detected. He recommended that the chromosomal karyotype and amniotic fluid AFP level be determined by amniocentesis at about 16 to 18 weeks. This should allow those pregnancies in whom the fetus is potentially normal to be selected for conservative managerment. ${ }^{17}$

Steller et al reporting on their experiences from the New England trophoblastic disease center (NETDC) have reported only eight well documented cases between 1973 and 1994 of which only one surviving fetus. All pregnancies were terminated between 14 and 21 weeks, seven out of eight had vaginal bleeding, 2 developed pre-eclampsia. ${ }^{18}$

It is very interesting that all of the reported cases of nontriploidy partial mole have had female karyotype (46 XX) except for one case reported by Teng and Ballon. ${ }^{9}$

Sulochana Gunasheela reported key points to reduce clinical dilemma in the management of partial molar pregnancy with coexistent viable fetus. ${ }^{19}$

1. Desire of woman to have a termination or continue pregnancy toward viability of a fetus has to be respected.

2. Decision cordocentesis in order to exclude triploidy.

3. Making responsibility certainly falls upon the clinician in attendance.

4. Age factor should be taken into consideration. Woman below 20 are $3 \frac{1}{2}$ times more at risk for postmolar diseases than those above 25 . Woman more than 40 years of age should be Diagnosis of normalcy of a fetus must be made either by amniocentesis or counseled in favor of a hysterectomy.

5. Obstetric complications should be considered in decision making.

\section{CONCLUSION}

Pregnancies with partial mole with coexistent live fetus is extremely rare because of numerous maternal and fetal complications including pre-eclampsia, hyperthyroidism, heavy vaginal bleeding, persistent gestational trophoblastic disease, preterm labor, fetal abortion and anemia.

Such a case presented to us at first stage of preterm labor and was managed successfully with team efforts of obstetrician, pediatrician and nursing staff.

In conclusion, partial molar pregnancy with a coexisting live fetus, in addition to chromosomal evaluation and detailed ultrasound examination to exclude fetal abnormality before the decision to postpone intervention, prenatal consultation should include complete discussion of maternal and fetal risks, especially the possible requirement of chemotherapy or even hysterectomy. It is also necessary to have close follow-up and evaluation of the condition of the patient during prenatal and postnatal period.

\section{REFERENCES}

1. Hsieh CC, Hsieh TT, Kuo DM, Lo LM, Hung TH. Delivery of a severely anaemic fetus after partial molar pregnancy: clinical and ultrasonographic findings. Hum Reprod 1999;14:11221126.

2. Agarwal R, Agarwal S, Roy KK. Diploid partial mole with neonatal survival: a case report. Indian J Pathol 2005 April;48:225-227.

3. Ohama K, Ueda K, Okamoto E, et al. Cytogenetic and clinicopathological studies of partial moles. Obstet Gynecol 1986; 68:259-266.

4. Guven ES, Ozturk N, Deveci S, Hizli D, Kandemir O, Dilbaz S. Partial molar pregnancy with coexisting fetus with diploid karyotype. J Matern Fetal Neonatal Med 2007 Feb;20(2):175181.

5. Sarno AP, Moorman AJ, Kalousek DK. Partial molar pregency with fetal survival: an unusual example of confined placental mosaicism. Obstet Gynecol 1993;82:716-719.

6. Jones WB, Lauersen NH. Hydatidiform mole with coexistent fetus. Am J Obstet Gynecol 1975;122:267-272.

7. Deaton JL, Hoffman JS, Saal H, et al. Molar pregnancy coexisting with a normal fetus: a case. Gynecol Oncol 1989;32:394-397. 
8. Crooiji MJ, Harten VD, PuyenbroekJJ, et al. A partial hydatidi-form mole dispersed throughout the placenta, coexisting with a normal living fetus: case report. Br J Obstet Gynaecol 1985;92:104-106.

9. Teng NNH, Ballon SC. Partial hydatidiform mole with diploid karyotype: report of three cases. Am J Obstet Gynecol 1984; 150:961-964.

10. Szulman, Surti. The syndrome of hydatiform mole (1) cytogenetic and morphological correlations. Am J Obstet Gynaecol 1978;68:259-266.

11. Parveen Z, Bashir R, Jadoon T, Qayum L. Partial hydatidiform mole along with term gestation and alive baby. J Ayub Med Coll Abbottabad 2004 Oct-Dec;16(4):84-85.

12. Pool R, Lebethe SJ, Lancaster EJ, Partial hydatidiform mole with a coexistent live full term fetus: a case report. S Afr Med J 1989 Feb 18;75(4):186-187.

13. Dhingra KK, Gupta P', Saroha V, et al. Partial hydatidiform mole with a full term infant. Indian J Pathology and Microbiology 2009; 52(4):590-591.
14. Vejerslev LO. Clinical management and diagnostic possibilities in hydatidiform mole with coexistent fetus. Obstet Gynecol Surv 1991;46:577-588.

15. Nwosu EC, Ferriman E, McCormack MJ. Partial hydatidiform mole and hypertension associated with a live fetus: variable presentation in two cases. Hum Reprod 1995;10:2459-2462.

16. Zahida P, Rubina B, Taimur J, Iftikhar Q, Partial hydatidiform mole along with term gestation and alive baby. J Ayub Med Call Dec 2004;16(4):84-85.

17. Khoo SK, Monks PL, Davies NT. Hydatidiform mole with coexistent live fetus cytogenetic features, diagnosis and management. Minerva Ginecol 2000 Jun;52(6):253-257.

18. Steller MA, Genest DR, Bernstein MR, et al. Natural history of twin pregnancy with complete hydatidiform mole and co-existing fetus. Obstel Gynaecol 1977;127-167.

19. Gunasheela S. Hydatidiform mole with a coexistent live fetus (HMCLF): a clinical dilemma. Singapore J Obstetrics and Gynecology 2001 July;32(2):10-17. 